Intensivmed 2011 · 48:174-174

DOI 10.1007/s00390-011-0281-y

Online publiziert: 7. April 2011

(c) Springer-Verlag 2011

\section{A. Geppert}

Herzintensivstation und Herzkatheterlabor, 3. Medizinische Abteilung mit

Kardiologie und internistischer Notaufnahme, Wilhelminenspital, Wien

\title{
Kardiopulmonale Reanimation
}

Die Intensivmedizin hat in den letzten Jahren entscheidende Schritte zu einer Qualitätsverbesserung gemacht, weniger durch bahnbrechende Erfindungen oder gar neue Medikamente, sondern indem kritisch reevaluiert und adaptiert wurde. Dogmen sind gefallen! So auch bei einer der ureigensten Domänen der Intensivmedizin, dem Herz-Kreislauf-Stillstand bzw. der Reanimation. Eine der letzten Errungenschaften ist die Kühlung nach Reanimation, während $z$. B. die Lysetherapie oder der Einsatz von Vasopressin nicht die erhofften Effekte gezeigt haben. Dennoch hat sich in der Behandlung von Patienten nach erfolgreicher Reanimation einiges getan!

\section{Neue ERC-Guidelines 2010}

Da 2010 ein Update der Leitlinien des European Resuscitation Councils (ERC) zur Reanimation erschienen ist, ist es an der Zeit, sich mit den darin enthaltenen Neuerungen auseinanderzusetzen. Aus diesem Grund beschäftigt sich der erste Beitrag in diesem Heft von Dr. Raaz, Prof. Buerke und Kollegen aus Halle eben mit jenen Neuerungen und mit allfälligen Unterschieden in den Leitlinien zwischen inner- und außerhospitaler Reanimation, nicht nur hinsichtlich Beatmung während Herzdruckmassage.

Dass ein akutes Koronarsyndrom (ACS) ein sehr häufiger Auslöser für einen Herz-Kreislauf-Stillstand ist, ist allgemein bekannt. In den neuen Leitlinien wird der Koronarangiographie und der Revaskularisation nach Reanimation großer Stellenwert eingeräumt; ebenso wurden sie hinsichtlich antithrombotischer Begleitmedikation an die Guidelines der European
Society of Cardiology (ESC) zum ST- und Nicht-ST-Hebungsinfarkt angepasst. Insbesondere wird darauf hingewiesen, dass eigentlich keine Unterschiede in der Indikationsstellung zur Koronarangiographie zwischen ACS bei Patienten nach erfolgreicher Reanimation und ACS ohne Kreislaufstillstand gemacht werden sollten. Es wird sogar ausdrücklich hervorgehoben, dass die Koronarangiographie bei Patienten mit Rückkehr des Spontankreislaufs (ROSC) nach Kreislaufstillstand Teil eines standardisierten Postreanimationsprotokolls sein könnte. Daher beschäftigt sich der zweite Beitrag in diesem Heft von Prof. Delle Karth aus Wien mit der perkutanen koronaren Intervention nach Kreislaufstillstand und erfolgreicher Reanimation.

Die Kühlung nach Reanimation gehört heutzutage zum Standardprotokoll nach ROSC, wenn auch bei manchen Patientengruppen mit niedrigerem Evidenzgrad als bei anderen. Gerade in Hinblick auf dringend notwendige Untersuchungen wie z. B. eine Koronarangiographie ist aber genau zu überlegen, mit welchen Modalitäten die Patienten nach ROSC gekühlt werden sollten. Der dritte Beitrag dieses Themenheftes von Prof. von Lewinski und Prof. Pieske aus Graz beschäftigt sich daher mit den Indikationsstellungen zur therapeutischen Hypothermie und ihren Modalitäten und damit auch mit so wichtigen Fragen wie allfälliger antipyretischer Begleitmedikation und den Effekten der therapeutischen Hypothermie bei Patienten mit Infarkt.

Ein vierter Beitrag (Dr. Hasper und Kollegen, Berlin) beschäftigt sich als thematisch passender, freier Beitrag mit der Prognose nach erfolgreicher Reanima- tion und der Verwertbarkeit bisheriger prädiktiver Parameter im „Zeitalter der Kühlung“.

Im Vertrauen, damit ein für Sie interessantes und thematisch stimulierendes Themenheft gestaltet zu haben, verbleibe ich mit herzlichem Dank an die Autoren

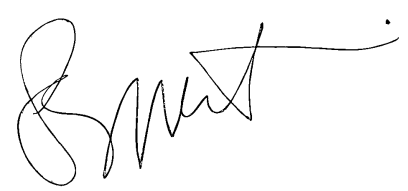

Ihr Alexander Geppert

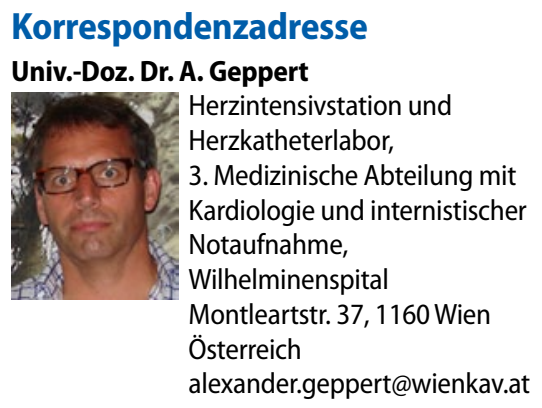

